8. When the patients were treated with an infusion of sodium bicarbonate or THAM which should induce elevation of $\mathrm{pH}$ and buffer base to a certain degree, a slight prolongation occurred only in the venous circulation. Compaired them with cases of the hyperventilation, there was a considerable difference in the arterial and capillary circulation, since the hyperventilation caused both circulations to be delayed.

\title{
g-2. Experimental Studies on Ligation of the Superior Sagittal Sinus
}

\author{
Junichi ShIraI, Fumiaki KaI, Nariaki Matuoka and Ikuzo Yokoyama \\ Ist Department of Surgery, School of Medicine, Kumamoto University
}

Using mongrel dogs, influence of ligation of the dorsal sagittal sinus on EEG and cerebrospinal fluid pressure was studied.

After the dorsal sagittal sinus was ligated at a site posterior to the cruciate sulcus or all tributary veins draining to the sinus were ligated, slow wave and low vo'tage activities were observed temporalily in EEG and the cerebrospinal fluid pressure measured slightly elevated.

In other $10 \mathrm{dogs}$, the sinus was ligated at a site posterior to the cruciate su'cus, 55 hours after a piece of laminaria was inserted into the extradural space to induce cerebral edema.

In 8 of the 10 dogs, an obvious abnormalities in EEG appeared 15-30 minutes after the ligation and continued for 70-100 minutes. An elevation of the cerebrospinal fluid pressure was also extensive.

Other dogs which were operated previously to induce edema in the same way as described above were injected with C.D.P. Cholin, Mannitol, Kallikrein or Prednisolone before or after ligation of the sinus and changes in EEF activities caused by the procedures were studied.

These drugs except Kallikrein showed noticeable effects on reducing the EEG abnormalities.

A ligation of the superior sagittal sinus in patients, in contrast to dogs, is sometimes followed by severe intracranial hypertension.

The drugs administered prior to ligation of the sinus might reduce further aggravation of cerebral damage due to acute venous stasis following ligation of the sinus. 